\title{
Two Chloroplast-Localized Proteins: AtNHR2A and AtNHR2B, Contribute to Callose Deposition During Nonhost Disease Resistance in Arabidopsis
}

\author{
Raksha Singh, ${ }^{1}$ Seonghee Lee, ${ }^{2}$ Laura Ortega, ${ }^{1}$ Vemanna S. Ramu, ${ }^{2}$ Muthappa Senthil-Kumar, ${ }^{2}$ \\ Elison B. Blancaflor, ${ }^{2}$ Clemencia M. Rojas, ${ }^{1,2,+}$ and Kirankumar S. Mysore ${ }^{2}$ \\ ${ }^{1}$ Department of Plant Pathology, University of Arkansas, Fayetteville, AR 72701, U.S.A.; ${ }^{2}$ Noble Research Institute, LLC., \\ Ardmore, OK 73401, U.S.A.
}

Accepted 5 June 2018.

\begin{abstract}
Plants are naturally resistant to most pathogens through a broad and durable defense response called nonhost disease resistance. Nonhost disease resistance is a complex process that includes preformed physical and chemical barriers and induced responses. In spite of its importance, many components of nonhost disease resistance remain to be identified and characterized. Using virus-induced gene silencing in Nicotiana benthamiana, we discovered a novel gene that we named $N b N H R 2$ ( $N$. benthamiana nonhost resistance 2). NbNHR2-silenced plants were susceptible to the nonadapted pathogen Pseudomonas syringae pv. tomato $\mathrm{T} 1$, which does not cause disease in wild-type or nonsilenced $N$. benthamiana plants. We found two orthologous genes in Arabidopsis thaliana: AtNHR2A and AtNHR2B. Similar to the results obtained in $N$. benthamiana, Atnhr2a and Atnhr2b mutants were susceptible to the nonadapted bacterial pathogen of $A$. thaliana, $P$. syringae pv. tabaci. We further found that these mutants were also defective in callose deposition. AtNHR2A and AtNHR2B fluorescent protein fusions transiently expressed in $N$. benthamiana localized predominantly to chloroplasts and a few unidentified dynamic puncta. RFP-AtNHR2A and AtNHR2BGFP displayed overlapping signals in chloroplasts, indicating that the two proteins could interact, an idea supported by coimmunoprecipitation studies. We propose that AtNHR2A and AtNHR2B are new components of a chloroplast-signaling pathway that activates callose deposition to the cell wall in response to bacterial pathogens.
\end{abstract}

Current address for Seonghee Lee: Gulf Coast Research and Education Center, University of Florida, Wimauma, FL 33598

Current address for Vemanna S. Ramu: Department of Crop Physiology, UAS, GKVK, Bangalore, India

Current address for Muthappa Senthil-Kumar: National Institute of Plant Genome Research, New Delhi, India

Funding: This work was supported by Noble Research Institute, LLC (Kirankumar S. Mysore) and by the start-up support from the University of Arkansas (Clemencia M. Rojas).

${ }^{\dagger}$ Corresponding author: Clemencia M. Rojas; E-mail: cr022@uark.edu

*The $\boldsymbol{e}$-Xtra logo stands for "electronic extra" and indicates that three supplementary figures and one supplementary table are published online.

(c) 2018 The American Phytopathological Society
Plants are exposed to a variety of pathogenic microbes during their life cycle and protect themselves through a complex innate immune system (Jones and Dangl 2006). The plant innate immune system includes preformed physical and chemical barriers that prevent pathogen ingress and, also, induce defenses that recognize the pathogen and limit its growth and proliferation (Senthil-Kumar and Mysore 2013; Lee et al. 2017). The combination of specific preformed barriers and induced responses in a given plant as well as the genetic makeup of the pathogen determine the specificity of the interaction between the plant and pathogen. A plant is considered a host if its genetic makeup provides a suitable niche for pathogen growth and, as a consequence of that, is susceptible to the pathogen and succumbs to pathogen infection. On the other hand, the plant is considered a nonhost if it is resistant to pathogen attack and restricts pathogen proliferation. All plants within the same species are resistant to certain pathogens; a phenomenon known as nonhost disease resistance (Heath 2000). A pathogen that has the ability to cause disease on a given plant is called a compatible or adapted pathogen, while a pathogen unable to cause disease in a given plant is called incompatible or nonadapted pathogen (Senthil-Kumar and Mysore 2013).

At early stages of plant-pathogen interactions, plants are able to detect a potential pathogen by recognizing broadly conserved molecular features on the pathogen surface known as PAMPs (pathogen-associated molecular patterns) in a process known as PTI (PAMP-triggered immunity) (Jones and Dangl 2006). This recognition is achieved by pathogen recognition receptors that contain extracellular domains (Boller and Felix 2009). Successful pathogens that are able to bypass PTI by the deployment of pathogen-specific proteins, known as effectors (Toruño et al. 2016), can be detected at later stages of the interaction by a second layer of immunity known as ETI (effector triggered immunity) (Jones and Dangl 2006). Pathogen recognition during ETI is mediated by intracellular nucleotidebinding-leucine-rich repeat (NLR) receptors (Khan et al. 2016). Both PTI and ETI lead to the activation of a signal transduction cascade involving reactive oxygen species (ROS), mitogen-activated protein kinases and hormones (Tsuda and Katagiri 2010). If the pathogen is unable to evade the plant immune system, the combined activities of PTI and ETI result in a strong defense response, with several outputs, that stops pathogen proliferation (Tsuda and Katagiri 2010).

One of the outputs of a plant defense response is the accumulation of callose (a $\beta-1,3$ glucan) in the paramural space, an area located between the plasma membrane and the cell wall (Luna et al. 2011). Deposition of callose at the cell wall is a 
common strategy that plants use in response to potential nematode, fungal, oomycete, and bacterial pathogens, and, because of its importance, it has been investigated for many years (Luna et al. 2011). Callose deposition has been presumed to provide strength to the cell wall to diminish pathogen entry. However, observations that mutants defective in callose synthase showed increased resistance to fungal pathogens (Nishimura et al. 2003; Vogel and Somerville 2000) appear to conflict with the proposed protective role of callose in plant defense. Callose accumulation is a complex process that is dependent on ROS (Galletti et al. 2008; Rojas et al. 2012), hormone signaling pathways (Luna et al. 2011; Nishimura et al. 2003; Ton and
Mauch-Mani 2004; Yi et al. 2014), as well as the indole glucosinolate biosynthetic pathway (Clay et al. 2009). The convergence of different pathways suggests complex regulatory mechanisms that are not fully understood (Luna et al. 2011).

Here, we report on the identification of NbNHR2 ( $N$. benthamiana nonhost resistance 2), a novel gene in Nicotiana benthamiana involved in nonhost disease resistance. Two NbNHR2 orthologs were found in Arabidopsis thaliana and were named AtNHR2A (Arabidopsis thaliana nonhost resistance 2A) and AtNHR2B (Arabidopsis thaliana nonhost resistance 2B). We showed that Arabidopsis mutants deficient in AtNHR2A and AtNHR2B are susceptible to the nonadapted pathogen of Arabidopsis,

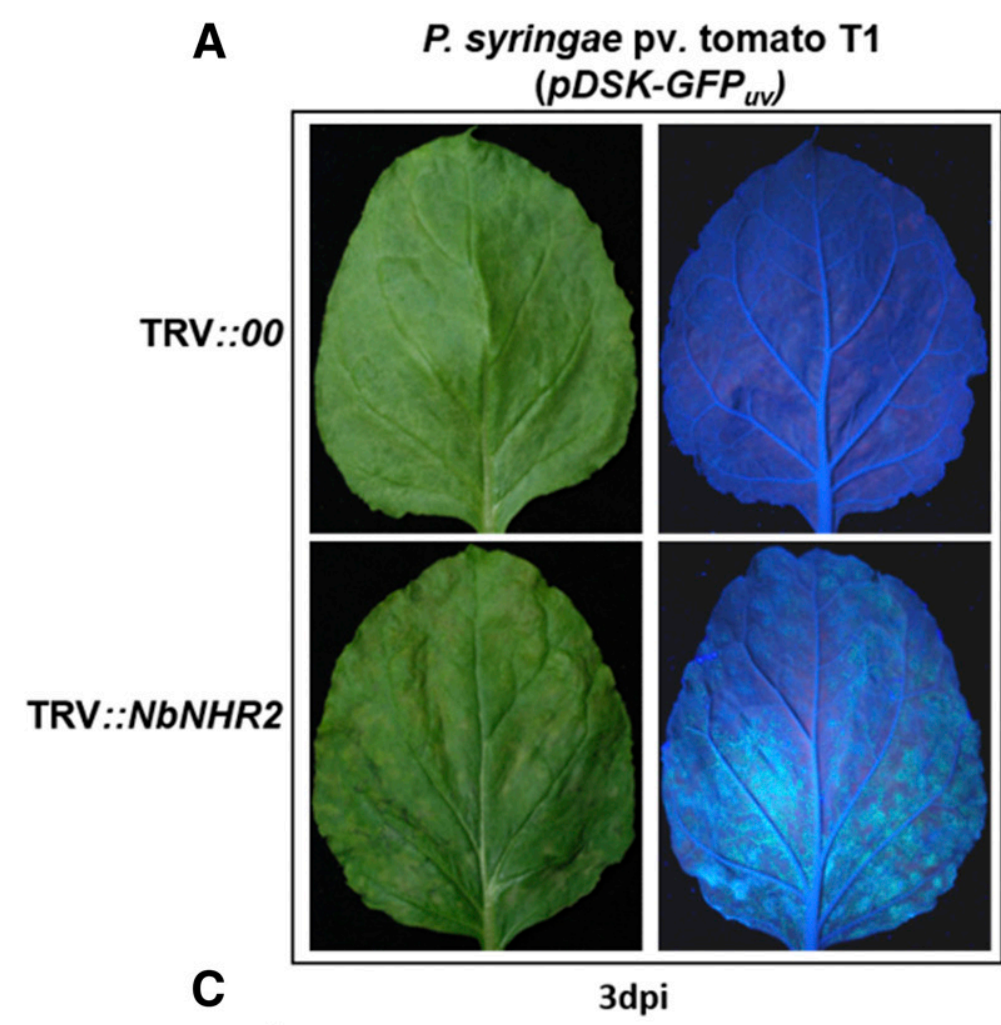

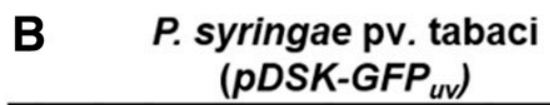
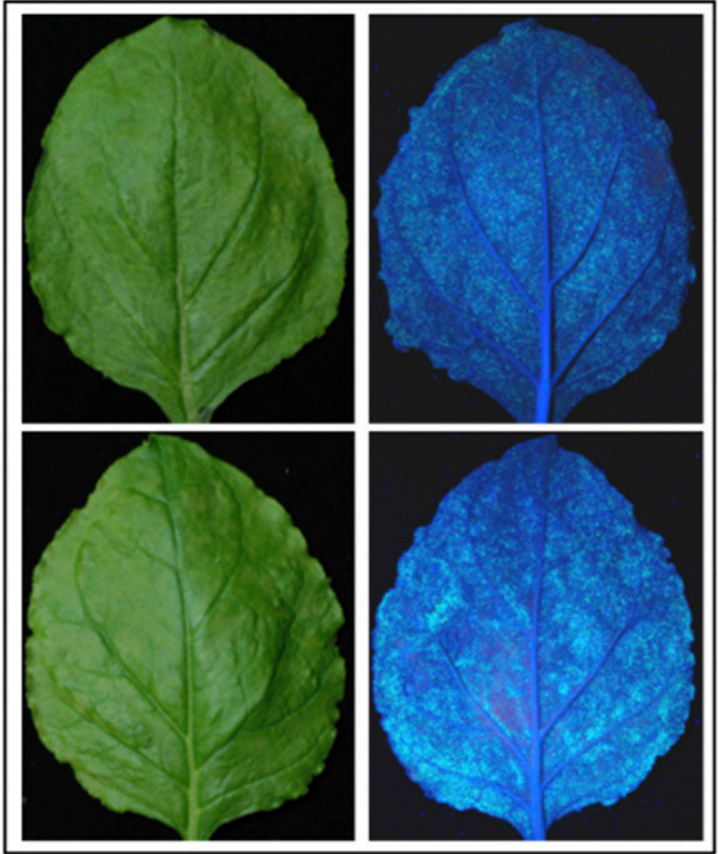

D

3dpi

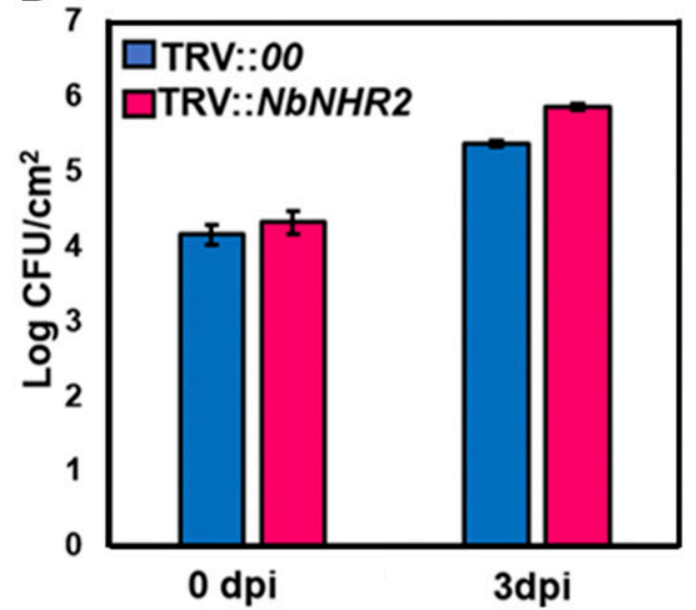

Fig. 1. NbNHR2 is involved in nonhost disease resistance in Nicotiana benthamiana. A and C, Virus-induced gene silencing was used to downregulate NbNHR2 expression. Three weeks after silencing, NbNHR2-silenced plants (TRV::NbNHR2) and nonsilenced controls (TRV::00) were vacuum-infiltrated with the nonadapted pathogen Pseudomonas syringae pv. tomato T1 ( $p D S K$-GFPuv) at $1 \times 10^{4} \mathrm{CFU} / \mathrm{ml}$ to observe the accumulation of fluorescence associated with bacterial growth after 3 days postinoculation (dpi) and to quantify bacterial populations at 0 and 3 dpi. B and D, Three weeks after silencing, NbNHR2-silenced plants (TRV::NbNHR2) and nonsilenced controls (TRV::00) were vacuum-infiltrated with the adapted pathogen $P$. syringae pv. tabaci $(p D S K$-GFPuv) at $1 \times$ $10^{4} \mathrm{CFU} / \mathrm{ml}$ to observe the accumulation of fluorescence associated with bacterial growth after 3 dpi and to quantify bacterial populations at 0 and 3 dpi. Bars represent mean and standard deviation of $P$. syringae pv. tomato T1 ( $p D S K$-GFPuv) and $P$. syringae pv. tabaci ( $p D S K$-GFPuv) populations in NbNHR2-silenced plants (TRV::NbNHR2) and nonsilenced controls (TRV::00). The asterisk represents statistically significant difference at $P<0.05$ between $T R V:: 00$ and $T R V::$ NbNHR2 based on Student's $t$ test for three biological replicates. All experiments were repeated at least three times with similar results. 
Pseudomonas syringae pv. tabaci, and are defective in callose deposition. We further showed that AtNHR2A and AtNHR2B colocalize to chloroplasts and interact with each other. We propose that AtNHR2A and AtNHR2B are new components of the chloroplast-mediated immune signaling pathway that mediates callose deposition in the cell wall in response to bacterial pathogens during nonhost disease resistance.

\section{RESULTS}

\section{NbNHR2 in Nicotiana benthamiana is involved in nonhost disease resistance.}

Our previous forward genetic screens using virus-induced gene silencing (VIGS) in Nicotiana benthamiana led to the identification of novel molecular players involved in nonhost disease resistance (Rojas et al. 2012; Wang et al. 2012). Here, we further characterized NbNHR2 ( $N$. benthamiana nonhost resistance 2) gene. NbNHR2 (locus ID LOC107776128) encodes a 346-amino acid protein of unknown function that is widely conserved in all plant species with available genomic sequences (Supplementary Fig. S2). Although VIGS caused 90\% downregulation of NbNHR2 transcripts (Supplementary Fig. S1), NbNHR2- silenced plants did not show any visible developmental phenotypes. To determine whether $N b N H R 2$ plays a role in nonhost disease resistance, $N b N H R 2$-silenced (TRV::NbNHR2) and control plants (TRV::00) were inoculated with the nonadapted pathogen $P$. syringae pv. tomato T1 expressing the green fluorescent protein (GFP) in plasmid $p D S K$ $G F P_{u v}$, which uses long wavelength ultraviolet light (Wang et al. 2007). As a nonadapted pathogen of $N$. benthamiana, $P$. syringae pv. tomato $\mathrm{T} 1$ does not cause successful infections in wild-type plants or in the nonsilenced controls (Fig. 1A). However, at 3 days postinoculation (dpi), we saw enhanced growth of $P$. syringae pv. tomato T1 in NbNHR2-silenced plants, observed as intense bacterial fluorescence (Fig. 1A). Quantification of bacterial growth in NHR2-silenced plants was approximately

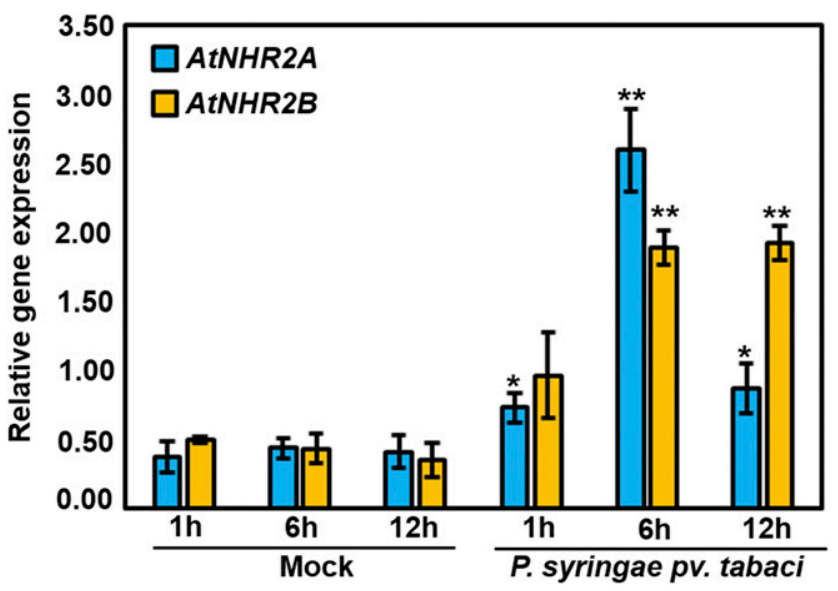

Fig. 2. AtNHR2A and AtNHR2B have distinct patterns of gene expression. Four-week-old wild-type Col-0 plants grown on $1 \times$ Murashige and Skoog plates were inoculated with Pseudomonas syringae pv. tabaci at $1 \times 10^{6}$ $\mathrm{CFU} / \mathrm{ml}$ and leaf samples were collected at 1, 6, and 12 hpi for RNA extraction and cDNA synthesis for real time quantitative reverse transcriptionpolymerase chain reaction, using AtNHR2A- and AtNHR2B-specific primers. $A t G A P D H$ (glyceraldehyde-3-phosphate dehydrogenase) was used as housekeeping gene for normalization. Expression of AtNHR2A or AtNHR2B in pathogen-inoculated plants was evaluated in comparison with mock-treated controls. Bars represent average and standard deviation for three biological replicates. Asterisks above bars represent statistically significant values when comparing pathogen-inoculated samples versus mock-inoculated controls for equivalent timepoints. One asterisk (*) indicates $P<0.01$, two (**) $P<0.001$. 100-fold higher than in nonsilenced controls (Fig. 1C). NbNHR2silenced and nonsilenced controls supported similar levels of bacterial multiplication after inoculation with the adapted pathogen $P$. syringae pv. tabaci (Fig. 1B, C, and D). These data indicate that downregulation of $N b N H R 2$ compromises nonhost disease resistance.

\section{AtNHR2A and AtNHR2B are Arabidopsis orthologs of $N b N H R 2$.}

Two NbNHR2 orthologs with loci IDs At4g25030 and At5g45410 were found in Arabidopsis thaliana and were named AtNHR2A and AtNHR2B, respectively. AtNHR2A is predicted to encode a 342 -amino acid protein, while $A t N H R 2 B$ is predicted to encode a 344 -amino acid protein. Both proteins have $65 \%$ amino acid sequence identity with each other and other regions of sequence similarity are found throughout the length of the proteins. BLASTP analysis identified closely related proteins to AtNHRA and AtNHR2B in monocots, dicots, and mosses. Phylogenetic analysis using closely related proteins identified from sequenced genomes showed that NbNHR2, AtNHR2A, and AtNHR2B belong to distinct clades (Saitou and Nei 1987). No significant domains to predict function were found for AtNHR2A or AtNHR2B.

\section{AtNHR2A and AtNHR2B have distinct patterns of gene expression and their respective mutants are compromised in nonhost disease resistance.}

To characterize the function of $A t N H R 2 A$ and $A t N H R 2 B$, we identified two transfer (T)-DNA insertion mutants in $A$. thaliana (Alonso and Stepanova 2003). SALK_114910 contains a T-DNA insertion in the third exon of At4g25030, and SALK_043140 contains a T-DNA insertion in the $5^{\prime}$ untranslated region of At5g45410 (Supplementary Fig. S3). Other T-DNA lines in Col-0 genetic background were not available. Homozygous Atnhr2a and Atnhr $2 b$ mutants were used to generate the double mutant Atnhr2b Atnhr2a.

Analysis of publicly available expression data using the Arabidopsis eFP browser (Winter et al. 2007) revealed that both AtNHR2A and AtNHR2B are expressed at very low levels during plant development and abiotic stress but they are significantly induced after treatment with diverse pathogens. We further analyzed the temporal patterns of gene expression for AtNHR2A and AtNHR2B after inoculation with $P$. syringae pv. tabaci and in comparison with mock-treated plants (Fig. 2). Induction of $A t N H R 2 A$ and $A t N H R 2 B$ was initiated at $1 \mathrm{~h}$ postinoculation (hpi). AtNHR2B expression progressively increased at 6 and $12 \mathrm{hpi}$, while AtNHR2A showed a peak of induction at $6 \mathrm{hpi}$ and its levels of expression sharply decreased at 12 hpi (Fig. 2). Taken together with publicly available expression data, our results suggest that $A t N H R 2 A$ and $A t N H R 2 B$ have distinct temporal patterns of gene expression after infection with $P$. syringae pv. tabaci.

To confirm that the Atnhr $2 a$ and Atnhr $2 b$ mutants are defective in expression of $A t N H R 2 A$ and $A t N H R 2 B$, respectively, we measured the expression of these genes, in their respective mutant backgrounds, by real time quantitative reverse transcription-polymerase chain reaction (qRT-PCR). Given that, in wild-type Col-0 plants, AtNHR2A and AtNHR $2 B$ are expressed at very low levels (Winter et al. 2007), we measured gene expression in wild-type Col-0, Atnhr2a, and Atnhr $2 b$ mutants after inoculation with $P$. syringae pv. tabaci. In comparison with wild-type Col-0 plants, we found that AtNHR $2 A$ expression in the Atnhr $2 a$ mutant was downregulated by $60 \%$, while the expression of AtNHR2B in the Atnhr $2 b$ mutant was downregulated by $50 \%$.

To investigate whether Atnhr2a and Atnhr2b mutants were compromised in disease resistance, we inoculated wild-type Col-0, the single mutants Atnhr2a and Atnhr2b, and the double 
mutant Atnhr2b Atnhr2a with the nonadapted Arabidopsis pathogen $P$. syringae pv. tabaci and the adapted pathogen $P$. syringae pv. tomato DC3000. As a nonadapted pathogen of Arabidopsis, $P$. syringae pv. tabaci did not cause disease symptoms in wildtype Col-0. On the other hand, it caused chlorosis in both Atnhr2a and $A t n h r 2 b$ and significant necrosis in the double mutant Atnhr2b Atnhr2a (Fig. 3A). Quantification of bacterial growth showed that, at $3 \mathrm{dpi}, P$. syringae pv. tabaci did not grow significantly in wild-type Col-0 when compared with the initial inoculum levels at 0 dpi. However, the populations of $P$. syringae pv. tabaci in the single mutants Atnhr2a and Atnhr2b were 10fold higher than in Col-0 (Fig. 3B). Significantly, P. syringae pv. tabaci growth at 3 dpi in the double mutant Atnhr $2 b$ Atnhr $2 a$ was approximately 100-fold higher than in either of the single mutants (Fig. 3B). Consistent with previous observations, the host pathogen $P$. syringae pv. tomato DC3000 caused disease symptoms in wild-type Col-0 plants and no significant differences in symptoms were observed due to this pathogen in either of the single-mutant plant genotypes (Fig. 3A). However, P. syringae pv. tomato DC3000 growth in the Atnhr2a Atnhr2b double mutants was 10-fold higher than wild type and single Atnhr $2 \mathrm{mu}-$ tants (Fig. 3C). These results indicate that AtNHR2A and AtNHR2B in Arabidopsis are involved in plant immunity against bacterial pathogens. The additive phenotype observed in the double mutant suggests that AtNHR2A and AtNHR2B have independent functions but act together in response to bacterial pathogens.

\section{AtNHR2A and AtNHR2B are involved in} callose deposition.

To dissect the function of $A t N H R 2 A$ and $A t N H R 2 B$, we decided to investigate whether these mutants were defective in callose deposition, a well-known marker of defense responses. For that purpose, wild-type Col-0, Atnhr2a, Atnhr2b, and
A

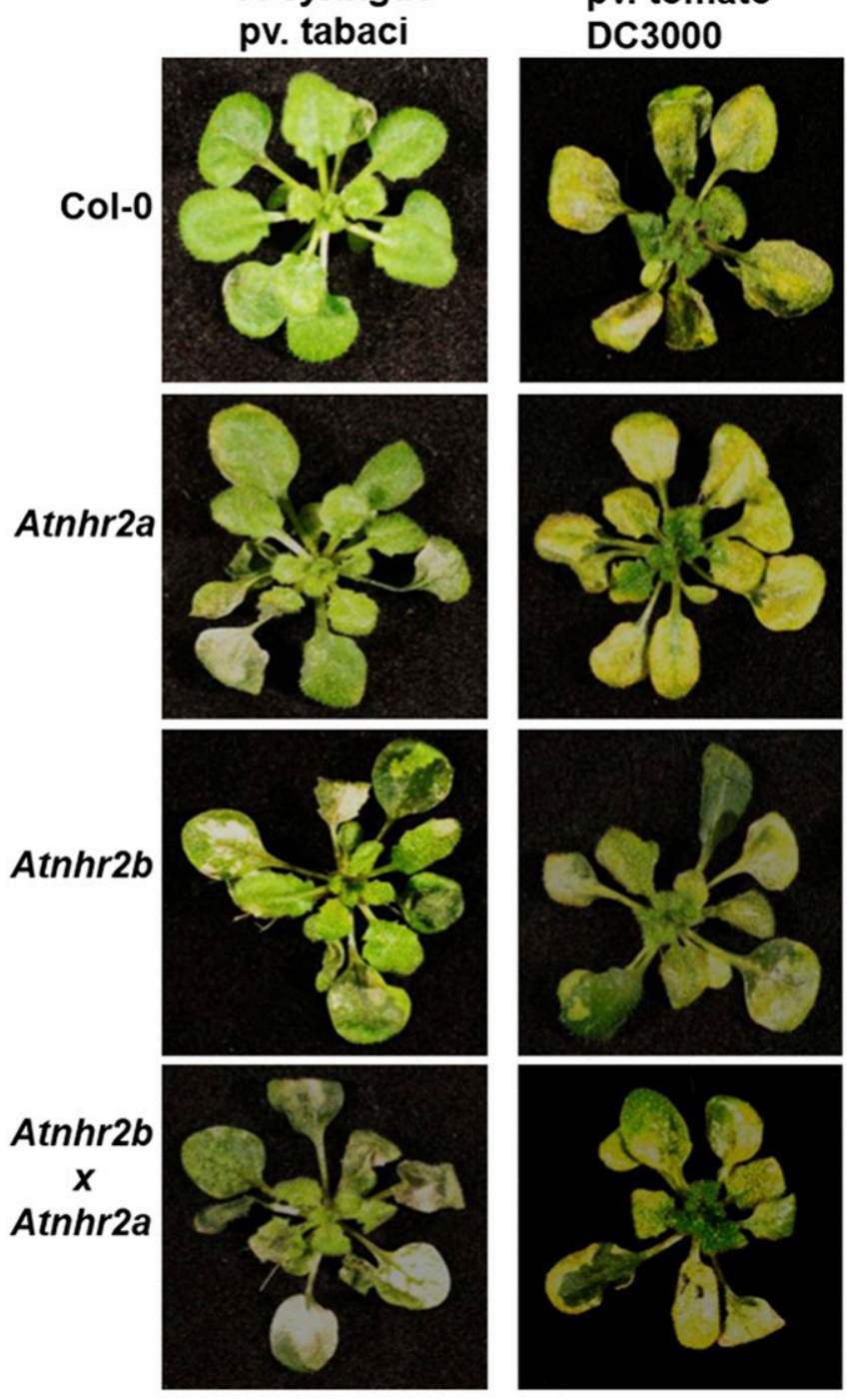
pv. tomato DC3000

\section{P. syringae}

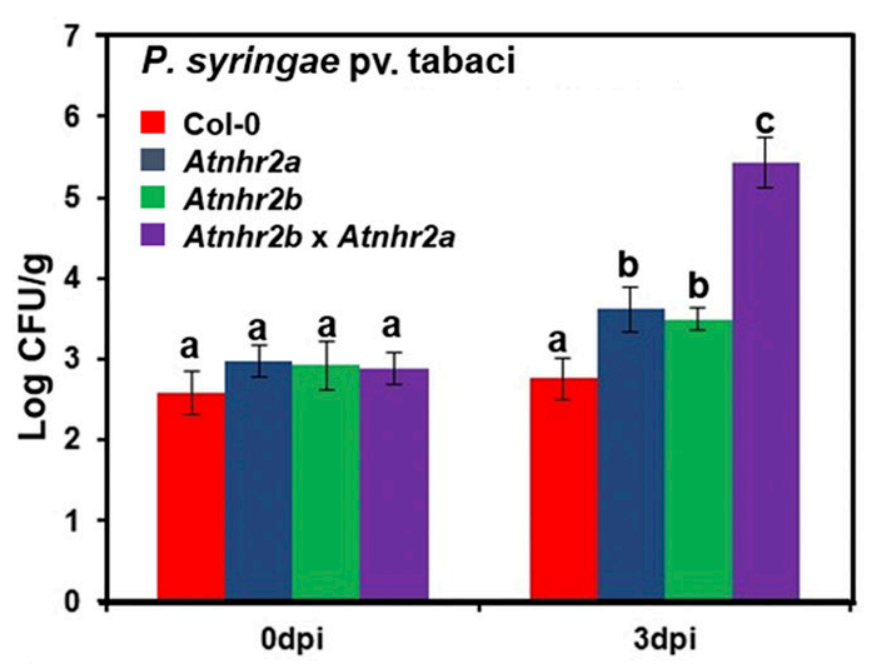

C

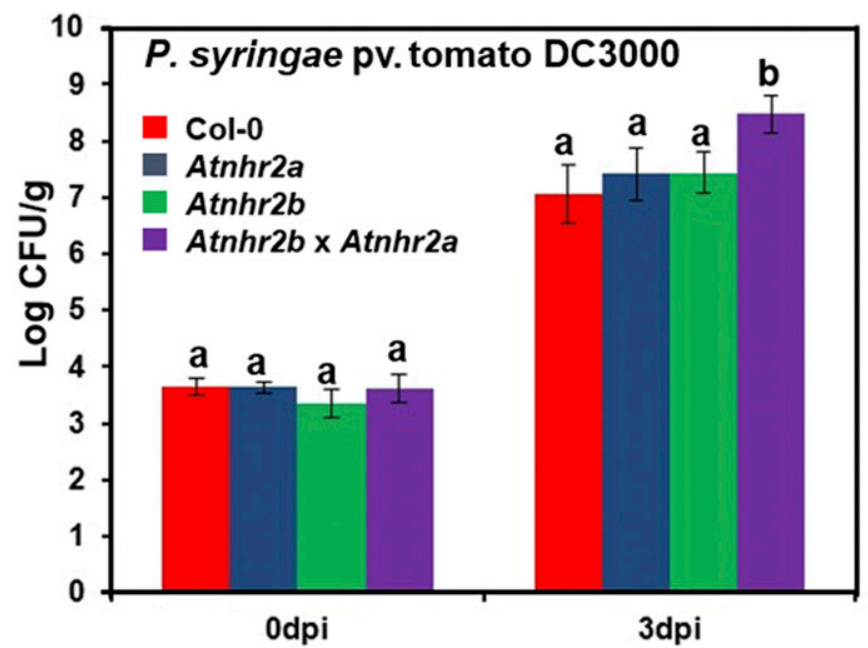

Fig. 3. Atnhr2a and Atnhr $2 b$ mutants are compromised in nonhost disease resistance and show enhanced disease symptoms. A, Disease symptoms in Arabidopsis thaliana. Four-week-old wild-type Col-0 and mutants Atnhr $2 a$, Atnhr $2 b$, and Atnhr $2 b$ Atnhr $2 a$ grown on $1 \times$ Murashige and Skoog plates were inoculated with the nonadapted pathogen Pseudomonas syringae pv. tabaci or the adapted pathogen $P$. syringae pv. tomato DC3000 at $1 \times 10^{4} \mathrm{CFU} / \mathrm{ml}$, to observe the development of symptoms after 5 days postinoculation (dpi). B and $\mathbf{C}$, Bacterial growth curves after pathogen inoculation. Inoculated plants were harvested and ground, were serially diluted, and were plated to enumerate bacterial populations at 0 and 3 dpi. Bars represent the mean and standard deviation for colony-forming units per gram per line per timepoint for three independent experiments. Means marked with the same letter are not statistically different based on Tukey's test $(P<0.05)$. 
Atnhr $2 b$ Atnhr $2 a$ mutants were inoculated with the nonadapted pathogen $P$. syringae pv. tabaci and leaves were detached and stained with aniline blue to observe callose deposits by confocal microscopy. As previously reported (Rojas et al. 2012), wild-type Col-0 showed abundant callose accumulation (approximately 100 callose deposits per microscopic field), but callose deposits were significantly reduced in both Atnhr2a and Atnhr $2 b$ mutants (Fig. 4). While Atnhr2a mutant had a $60 \%$ reduction in callose deposits as compared with Col-0, Atnhr $2 b$ mutant was almost completely depleted of callose deposits corresponding to about a $96 \%$ reduction in comparison with Col-0 (Fig. 4). Atnhr2b Atnhr2a double mutants had a phenotype similar to the Atnhr $2 b$ single mutant. Altogether, our data show that both $A t N H R 2 A$ and $A t N H R 2 B$ are involved in callose deposition, with AtNHR $2 B$ playing a more prominent role than AtNHR2A. The fact that the phenotype of the Atnhr2b Atnhr $2 a$ double mutant resembles the phenotype of the Atnhr $2 b$ single mutant suggests that $A t N H R 2 B$ and $A t N H R 2 A$ function in overlapping genetic pathways.

\section{AtNHR2A and AtNHR2B colocalize to the chloroplast} envelope and interact with each other.

To better understand the role of NHR2 in the disease phenotypes we observed, we studied the localization of AtNHR2A and AtNHR2B fluorescent protein fusions, using confocal microscopy. AtNHR2A and AtNHR2B contain a chloroplastic

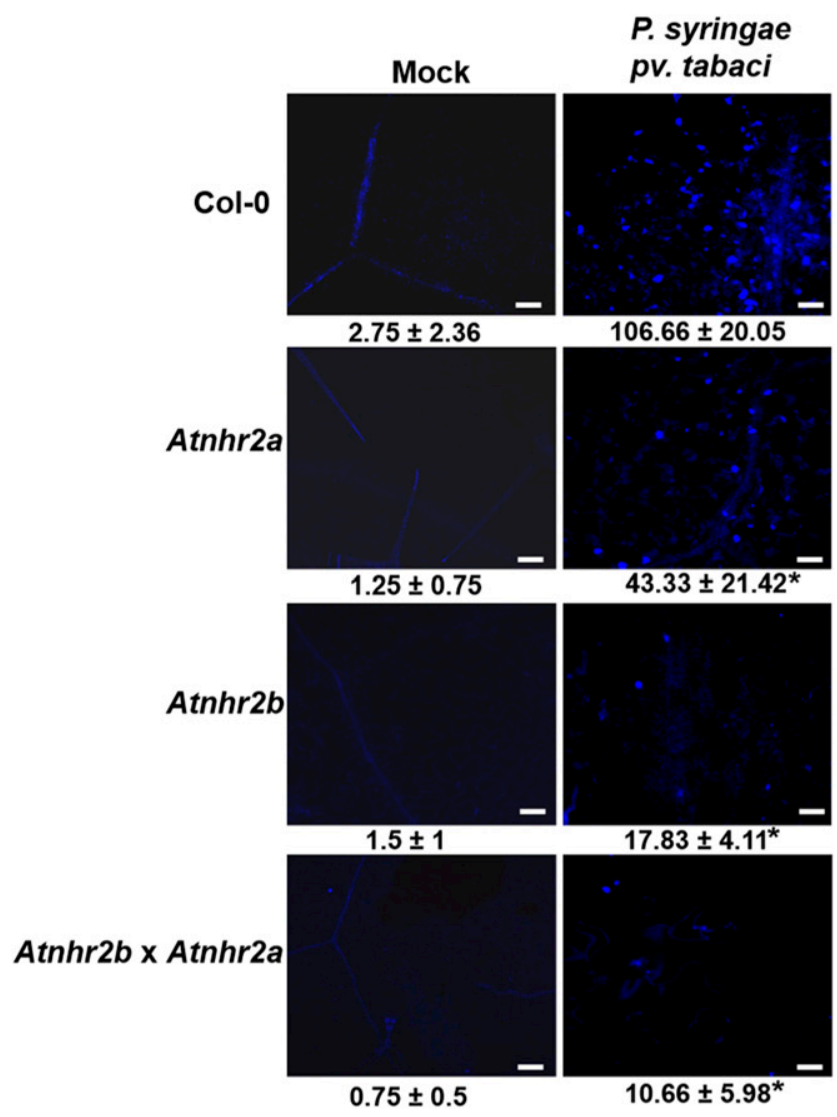

Fig. 4. AtNHR2A and $A t N H R 2 B$ are important in callose deposition. Fourweek-old plants were syringe-inoculated with the nonadapted pathogen $P$. syringae pv. tabaci at $1 \times 10^{6} \mathrm{CFU} / \mathrm{ml}$ or infiltrated with water and used as mock control. Leaves were harvested after $24 \mathrm{~h}$, were stained with $5 \%$ aniline blue, and were visualized by scanning laser confocal microscope using an excitation wavelength of $405 \mathrm{~nm}$ and an emission wavelength of 450 to $510 \mathrm{~nm}$. Numbers below the pictures indicate numbers of callose deposits for six random microscopic fields obtained from three independent experiments. Asterisks represent statistically significant value at $P<0.001$. Scale bar $=20 \mu \mathrm{m}$. targeting signal (Schwacke et al. 2007), as predicted by the publicly available database Aramemnon (Schwacke et al. 2003). Additional databases, such as ChloroP 1.1 (Emanuelsson et al. 1999), PredSL (Petsalaki et al. 2006), and Target $P$ (Emanuelsson et al. 2000), predicted several chloroplastic localization signals throughout the protein. While we do not know exactly which of those signals actually target AtNHR2A and AtNHR2B to the chloroplast, our biological data supports the prediction. We found that AtNHR2A-GFP and AtNHR2B-GFP predominantly overlapped with the chloroplast autofluorescence, indicating that these proteins are associated with chloroplasts (Fig. 5A and B). High magnification images revealed that both AtNHR2A-GFP and AtNHR2B-GFP signal surrounded the chloroplasts, indicating that they are localized to the chloroplast envelope (Fig. 5C and D).

We also found that AtNHR2A-GFP and AtNHR2B-GFP localized to small puncta reminiscent of membrane-bound organelles of the endomembrane system (Fig. 5A and B, long arrows), which includes a plethora of subcellular compartments, many of which remain to be characterized (Fujimoto and Ueda 2012). While we cannot specifically identify the localization of AtNHR2A-GFP and AtNHR2B-GFP to one or more particular compartments, future experiments using fluorescent markers labeling each one of those compartments (Geldner et al. 2009; Nelson et al. 2007) will enable us to do so.

The localization of AtNHR2A-GFP and AtNHR2B-GFP motivated us to investigate the possibility that both proteins colocalize, which would give us insight into their interdependent function. To be able to visualize both proteins simultaneously, we generated an AtNHR2A N-terminal fusion to the red fluorescent protein (RFP). Transient expression of RFP-AtNHR2A in N. benthamiana showed the same pattern of localization as AtNHR2A-GFP, indicating that the N-terminal tag does not interfere with the localization of the protein. Interestingly, transient coexpression in $N$. benthamiana of AtNHR2B-GFP and RFP-AtNHR2A showed colocalization of both proteins to the chloroplasts, as demonstrated by the overlap of the green and red signals (Fig. 5E).

The colocalization of AtNHR2B-GFP and RFP-AtNHR2A prompted us to hypothesize that both proteins interact to perform a common function. We therefore generated a protein fusion of AtNHR2A to the hemagglutinin (HA) tag and transiently coexpressed it in $N$. benthamiana with either AtNHR2B-GFP or 35S-GFP for coimmunoprecipitation with anti-HA antibodies conjugated to agarose beads. AtNHR2AHA coimmunoprecipitated AtNHR2B-GFP but not free GFP as detected by Western blot using anti-GFP antibodies (Fig. 6).

AtNHR2A and AtNHR2B fusions to GFP are functional.

To determine whether the observed chloroplast localization of the AtNHR2-GFP fusions represents the correct compartment that confers protein functionality, we transformed the Atnhr $2 a$ and Atnhr $2 b$ mutants with their respective GFP fusions and evaluated disease-related phenotypes. Wild-type Col-0, Atnhr2a mutant, and the complementing lines AtNHR2AGFP/Atnhr2a-2 and AtNHR2A-GFP/Atnhr2a-4 were syringeinoculated with the nonadapted bacterial pathogen $P$. syringae pv. tabaci to examine disease symptoms, bacterial growth, and callose deposition (Fig. 7A, B, and C). Whereas the Atnhr2a mutant showed severe disease symptoms, supported approximately 10-fold higher bacterial populations than wild-type Col-0, and was significantly reduced in callose accumulation, the complementing lines AtNHR2A-GFP/Atnhr2a-2 and AtNHR2AGFP/Atnhr $2 a-4$ did not exhibit disease symptoms. Bacterial populations on these lines were similar to bacterial populations in wild-type Col-0 plants and callose deposition was restored up to $50 \%$ levels (Fig. 7A, B, and C). Similarly, the AtNHR2BGFP/Atnhr2b-2 and AtNHR2B-GFP/Atnhr $2 b-4$ exhibited similar 
phenotypes as wild type with regard to bacterial growth curves and callose deposition (Fig. 8A, B, and C). Taken together, our results indicate that disease phenotypes of Atnhr $2 a$ and Atnhr $2 b$ are due to downregulation of $A t N H R 2 A$ and $A t N H R 2 B$, respectively, and that the AtNHR2-GFP fusions are functional.

We next asked whether AtNHR2A-GFP and AtNHR2B-GFP exhibited similar chloroplast localization as transient expression assays. Although AtNHR2B-GFP was able to complement the $A t n h r 2 b$ phenotypes, we were unable to detect GFP fluorescence in Atnhr $2 b$ lines expressing AtNHR $2 B-G F P$. It is possible that sufficient levels of AtNHR2B-GFP expression were present to rescue Atnhr $2 b$ phenotypes but not enough to be detectable with confocal microscopy (Moore and Murphy 2009). On the other hand, AtNHR2A-GFP in the Atnhr2a background localized predominantly to chloroplasts similar to observations in transient expression assays (Fig. 9A, B, and C).

\section{DISCUSSION}

Nonhost disease resistance has been investigated for many years due to its potential to be harnessed for crop improvement and given its durability and broad spectrum (Lee et al. 2016).

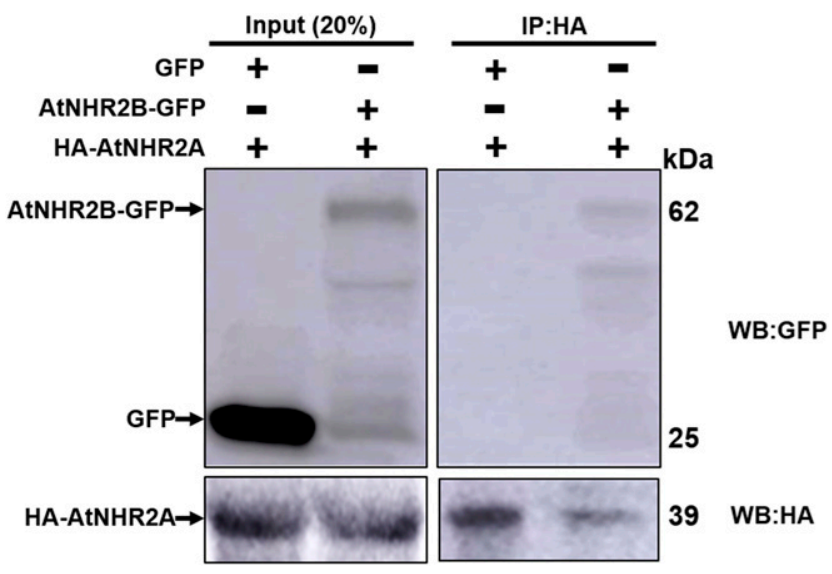

Fig. 6. AtNHR2A interacts with AtNHR2B. HA-AtNHR2A and either AtNHR2B-GFP or 35S-GFP were transiently coexpressed in Nicotiana benthamania leaves for coimmunoprecipitation with hemagglutinin (HA) antibodies conjugated to agarose beads. Expression of the proteins in the input fraction and their presence in the coimmunoprecipitated fraction were detected by Western blot using anti-green fluorescent protein (GFP) and anti-HA antibodies.
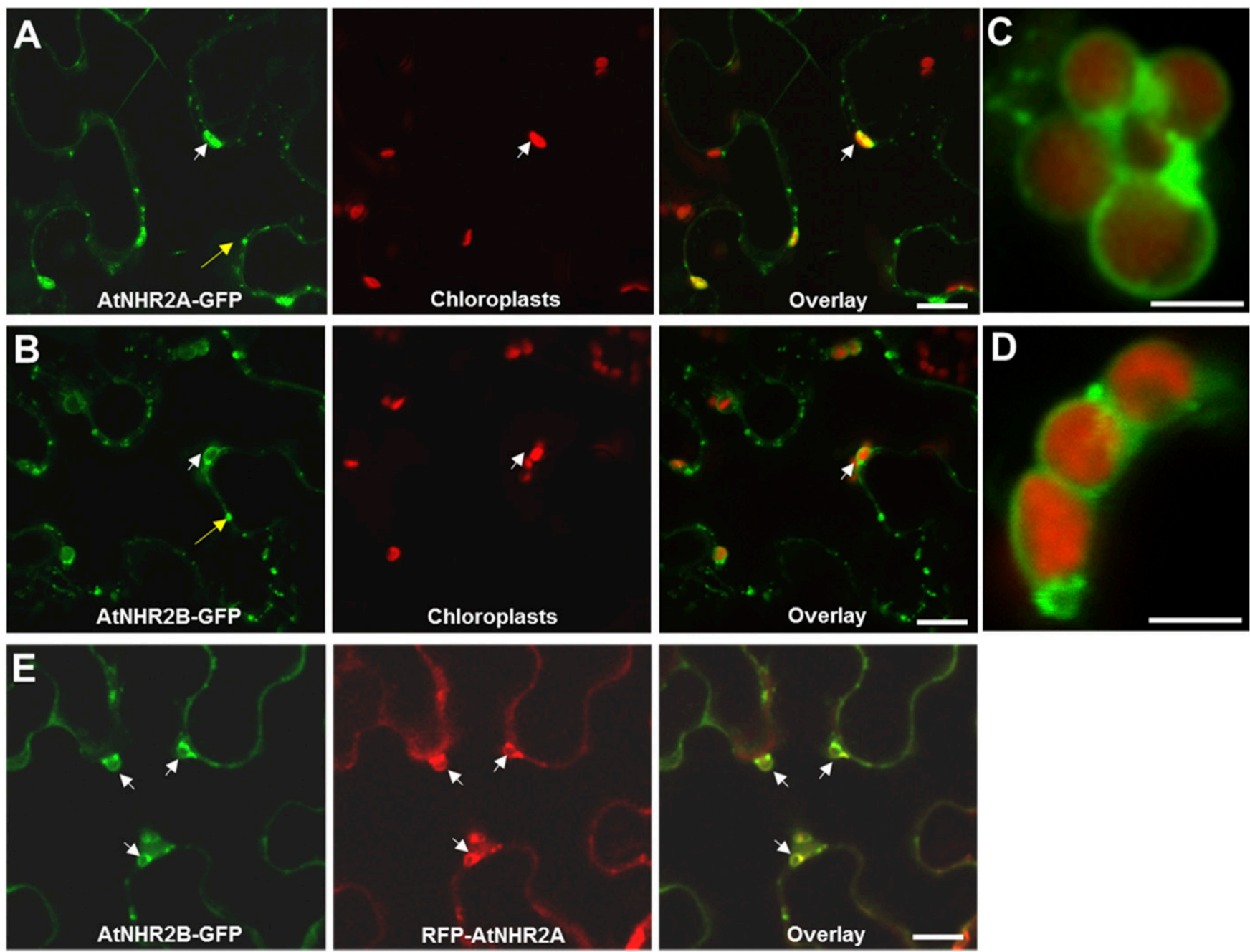

Fig. 5. AtNHR2A and AtNHR2B colocalize to chloroplasts. Three-week-old Nicotiana benthamiana plants were infiltrated with Agrobacterium tumefaciens strains harboring A, AtNHR2A-GFP and $\mathbf{B}, A t N H R 2 B-G F P$ constructs and were imaged by laser scanning confocal microscopy. Short arrows show that green fluorescent protein emission overlaps with chlorophyll autofluorescence. Long arrows in A and B show localization of AtNHR2A-GFP and AtNHR2B-GFP to puncta of unknown identity. C, Higher magnification images show that AtNHR2A-GFP and D, AtNHR2B-GFP signal is confined to the periphery of the chloroplasts. E, Three-week-old $N$. benthamiana plants were infiltrated with Agrobacterium tumefaciens strains harboring AtNHR2B-GFP and RFPAtNHR2A constructs. White arrows indicate overlapping of GFP and red fluorescent protein signals. Scale bar $=20 \mu \mathrm{m}$. 
However, despite its significance, mechanisms underlying nonhost resistance are still not fully understood. Specifically, it remains to be determined how nonhost disease resistance is integrated with other components of plant immunity. In this work, we identified NbNHR2, a gene in $N$. benthamiana that encodes an uncharacterized component of chloroplast-mediated nonhost resistance. Downregulation of $N b N H R 2$ by VIGS allowed a nonadapted pathogen of $N$. benthamiana, $P$. syringae pv. tomato $\mathrm{T} 1$, to cause disease and substantially proliferate in $N b N H R 2$ downregulated lines (Fig. 1). NbNHR2 is broadly conserved in plants and two orthologous genes (AtNHR2A and AtNHR2B) were identified in A. thaliana. AtNHR2A and AtNHR2B have $65 \%$ identity with each other at the amino acid sequence level, which indicates that these two proteins probably share some common functions. Although we were unable to infer the function of NHR2B based on putative domains of the proteins, the pathogen and cell biological assays presented here suggest new insights into the role of these two novel proteins in the context of plant immunity.

The connection between NHR2 and nonhost resistance is supported by two key observations. First, gene expression analysis using publicly available databases showed that AtNHR2A and $A t N H R 2 B$ have very low levels of expression during development but are induced in the presence of potential pathogens of diverse lifestyles, such as the fungus Botrytis cinerea, the adapted pathogen $P$. syringae pv. tomato DC3000, the avirulent bacterial pathogen $P$. syringae pv. tomato DC3000 (avrRpm1), the nonadapted pathogen $P$. syringae pv. phaseolicola, and the oomycete pathogen Phytophthora infestans. Both genes were also shown to be induced after treatment with elicitors derived from bacteria (flagellin and harpin) and oomycete pathogens (NPP1 [necrosis-inducing Phytophthora protein 1]). By inoculating A. thaliana wild-type Col-0 plants with the nonadapted pathogen $P$. syringae pv. tabaci, we further showed that each gene has a distinct temporal pattern of expression (Fig. 2), suggesting that these two genes might operate at different times after bacterial infection, despite being involved in similar processes. Second, both Atnhr2a and Atnhr2b were compromised in nonhost disease resistance as they supported successful infections of bacterial strains that normally are ineffective in wild-type plants (Fig. 3). We propose that compromised nonhost disease resistance in Atnhr2a and Atnhr $2 b$ is due, in part, to reduced callose accumulation (Fig. 4). Similar to our results, other Arabidopsis mutants that are defective in callose deposition are more susceptible to bacterial pathogens (De Benedictis et al. 2018; Rojas et al. 2012). Moreover, citrus plants downregulated in the callose synthase (CalS1) gene showed increased susceptibility to the bacterial pathogen Xanthomonas citri subsp. citri (Enrique et al. 2011). However, reduced callose accumulation does not always translate into enhanced disease susceptibility. For example, powdery mildew resistance 4 (pmr4) mutants, which are defective in the synthesis of pathogen-induced callose synthase, were resistant to the fungal and oomycete pathogens Erysiphe cihoracearum and Peronospora parasitica, respectively, but not to the bacterial pathogen $P$. syringae (Jacobs et al. 2003; Nishimura et al. 2003; Vogel and Somerville 2000). Although additional studies will be required to determine the precise relationship between callose accumulation and progression of bacteria-induced disease symptoms, our data indicate that

A
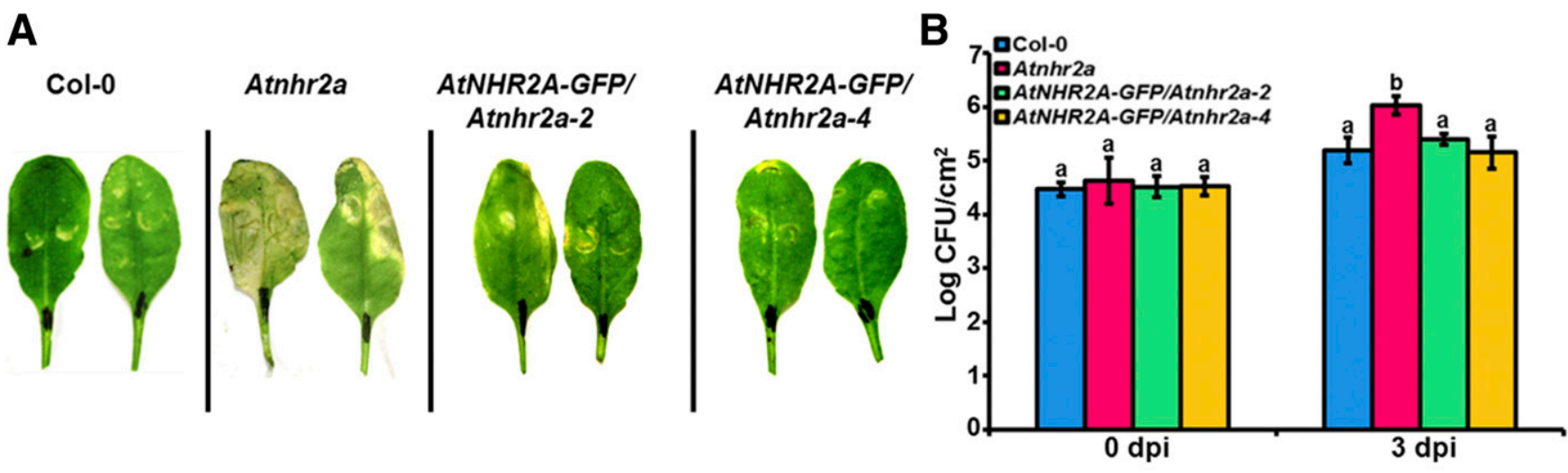

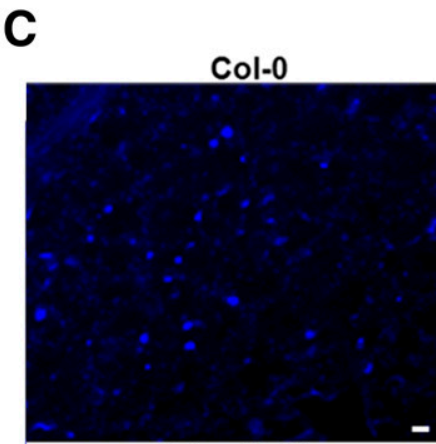

$104 \pm 30.19$

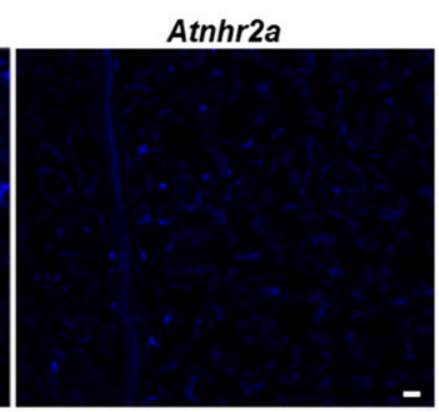

$15.5 \pm 4.13$

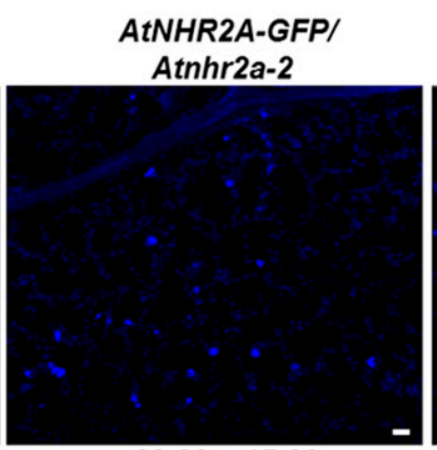

$62.83 \pm 15.09$

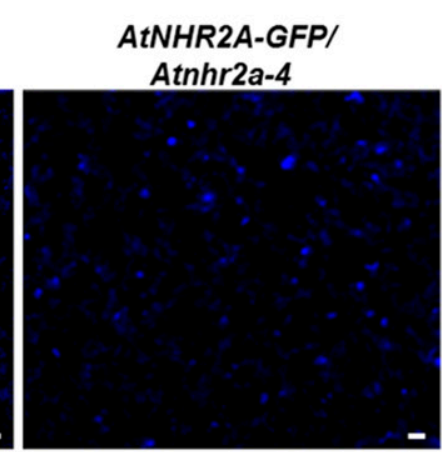

$79.33 \pm 18.35$

Fig. 7. Arabidopsis thaliana Atnhr $2 a$ mutation is complemented with a plasmid expressing AtNHR2A fused to green fluorescent protein (GFP). A to C, Fourweek-old wild-type Col-0, the mutant Atnhr2a, and two independent complemented lines, namely, AtNHR2A-GFP/Atnhr2a-2 and AtNHR2A-GFP/Atnhr2a-4, were syringe-inoculated with Pseudomonas syringae pv. tabaci at $1 \times 10^{6} \mathrm{CFU} / \mathrm{ml}$ to observe symptoms development (A), to monitor bacterial populations at 0 and 3 days postinoculation (dpi) (B), and to observe callose accumulation after aniline blue staining (C). Bars in B represent the mean and standard deviation for colony-forming units per square centimeter per line per timepoint for four biological replicates in three independent experiments. Means marked with the same letter are not statistically different, based on Tukey's test $(P<0.05)$. Numbers below the pictures in $\mathrm{C}$ indicate numbers of callose deposits for six microscopic fields obtained from three independent experiments. 
reduced callose is strongly correlated with the loss of nonhost resistance in NHR2 downregulated lines.

Although AtNHR2A and AtNHR2B appear to be induced at different times, the genetic, biochemical, and cell biological data we obtained here support the notion that the Arabidopsis NHR2 orthologs have overlapping functions. Independent downregulation of each gene led to a common output (i.e., reduced callose deposition) and the Atnhr2aAtnhr $2 b$ double mutant exhibited enhanced disease susceptibility. Functional overlap can also be inferred from the observation that AtNHR2A-GFP and AtNHR2B-GFP colocalized to the chloroplasts (Fig. 5E) and that AtNHR2A interacts with AtNHR2B as shown by coimmunoprecipitation (Fig. 6), although we do not yet know if that interaction is direct or occurs through other proteins forming a complex.

Callose accumulation in response to pathogens or pathogenderived elicitors is dependent on ROS (Galletti et al. 2008; Rojas et al. 2012), the abscisic acid (ABA), ethylene, salicylic acid (SA), and jasmonic acid (JA) signaling pathways (Luna et al. 2011; Nishimura et al. 2003; Ton and Mauch-Mani 2004; Yi et al. 2014), as well as the indole glucosinolate biosynthetic pathway (Clay et al. 2009). Because these molecules are synthesized in different subcellular compartments and sometimes through distinct metabolic pathways, it is still not clear how all these signals are integrated during callose biosynthesis, accumulation, and deposition. The finding that AtNHR2A and AtNHR2B are required for callose deposition and that AtNHR2A and AtNHR2B are associated with chloroplasts suggests that these proteins could be components of a chloroplast-mediated signaling pathway to activate defense responses after bacterial inoculation.
Establishing how AtNHR2A and AtNHR2B participate in chloroplast signaling during nonhost disease resistance, specifically as it relates to callose deposition, is not a trivial matter, given that chloroplasts are the source of important defense signaling molecules such as ROS and the hormones ABA, JA, and SA (Serrano et al. 2016), all of which have been implicated in callose deposition. Moreover, chloroplast-produced molecules are involved in the chloroplast retrograde pathway, a mechanism for chloroplast signals to regulate transcription of nuclear encoded genes (de Souza et al. 2017; Leister 2012). The chloroplast retrograde pathway is essential in plant development (de Souza et al. 2017) and, it has also been implicated in plant immunity. The chloroplast-localized CAS (calcium sensing receptor) protein that regulates SA biosynthesis in the chloroplast is also required for callose deposition and transcriptional activation of nuclear encoded genes in response to a bacterial PAMP, flagellin 22 (flg22) (Nomura et al. 2012). Furthermore, retrograde signaling mutants are compromised in the JA- and SA-signaling pathways and in the accumulation of indole glucosinolates in response to the adapted pathogen P. syringae pv. tomato DC3000 and the necrotrophic bacterial pathogen Pectobacterium carotovorum subsp. carotovorum EC1 (Ishiga et al. 2017). Retrograde pathways imply interorganellar communication, although the molecular mechanisms are only starting to be elucidated (de Souza et al. 2017), e.g., a protein localized to the chloroplast envelope and called PTM (plant homeodomain transcription factor with transmembrane domains) was shown to be cleaved and its N-terminus, containing the DNA binding, was found in the nucleus, in which it activated gene transcription (Sun et al. 2011). Interestingly,
A

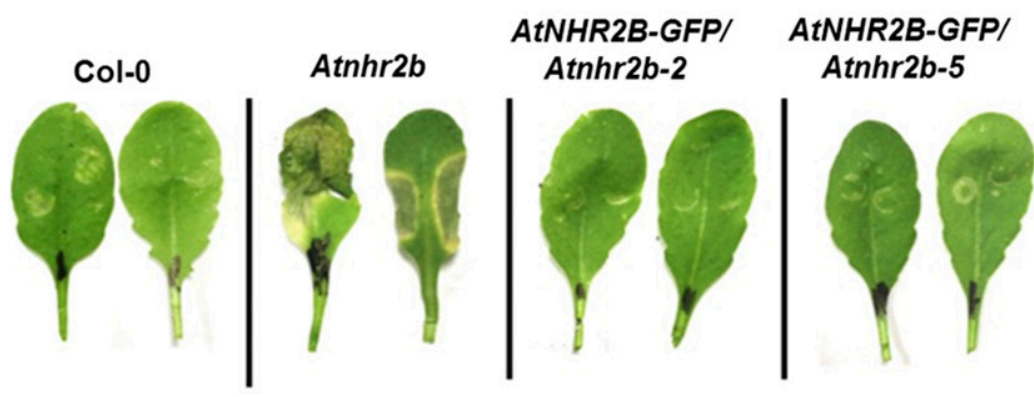

B

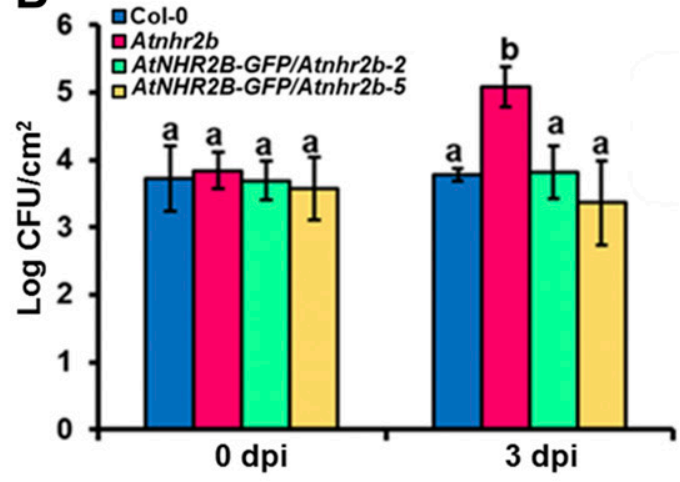

C

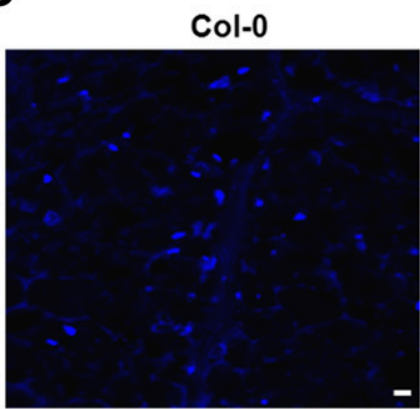

$98.2 \pm 9.25$

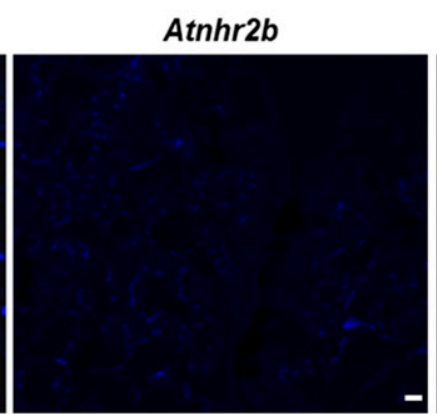

$7.2 \pm 1.64$
AtNHR2B-GFP/ Atnhr2b-2

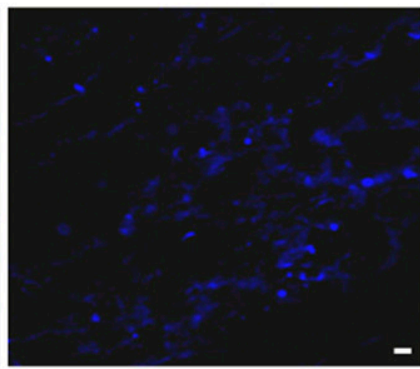

$60.4 \pm 5.17$
AtNHR2B-GFP/ Atnhr2b-5

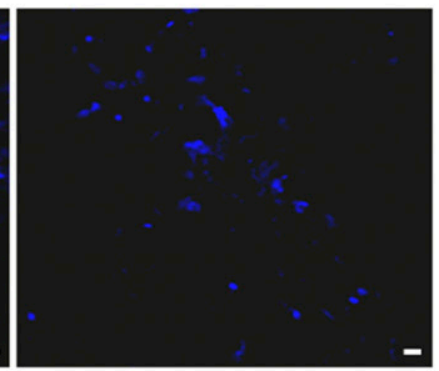

$64.60 \pm 10.16$

Fig. 8. Arabidopsis thaliana Atnhr $2 b$ mutation is complemented with a plasmid expressing AtNHR2B fused to green fluorescent protein (GFP). A to C, Fourweek-old wild-type Col-0, the mutant Atnhr $2 b$, and two independent complemented lines, namely, AtNHR2B-GFP/Atnhr2b-2 and AtNHR2B-GFP/Atnhr2b-5, were syringe-inoculated with Pseudomonas syringae pv. tabaci at $1 \times 10^{6} \mathrm{CFU} / \mathrm{ml}$ to observe symptom development (A), to monitor bacterial populations at 0 and 3 days postinoculation (dpi) (B), and to observe callose accumulation after aniline blue staining (C). Bars in B represent the mean and standard deviation for colony-forming units per square centimeter per line per timepoint for four biological replicates in three independent experiments. Means marked with the same letter are not statistically different, based on Tukey's test $(P<0.05)$. Numbers below the pictures in $\mathrm{C}$ indicate numbers of callose deposits for five microscopic fields obtained from three independent experiments. 
tubular structures derived from the chloroplast envelope and called stromules are formed after infection with Tobacco mosaic virus (Caplan et al. 2008) and P. syringae pv. tomato DC3000 expressing the effector proteins AvrRpm1, AvrRpt2, and AvrRps4 (Caplan et al. 2015). Stromules were observed connecting chloroplasts and nuclei, presumably to transport chloroplast-derived signals or proteins to the nucleus (Caplan et al. 2015).

In summary, we report on the identification of AtNHR2A and AtNHR2B, two previously undescribed chloroplast-localized proteins that play a role in nonhost disease resistance. Our results indicate that AtNHR2A and AtNHR2B are components of the chloroplast envelop functioning in the signaling machinery, leading to callose deposition during exposure to nonadapted bacterial pathogens. While it is known that chloroplasts are fundamental in plant immunity (Nomura et al. 2012), it is not clear how they participate in callose deposition during nonhost disease resistance. The discovery of the NHR2 proteins pave the way toward a more mechanistic understanding of this process.

\section{MATERIALS AND METHODS}

\section{Bacterial strains.}

Pseudomonas syringae strains were cultured in King's B medium, at $30^{\circ} \mathrm{C}$, supplemented with rifampicin $(25 \mu \mathrm{g} / \mathrm{ml})$. Agrobacterium tumefaciens strains were cultured at $28^{\circ} \mathrm{C}$ in Luria-Bertani medium supplemented with rifampicin $(25 \mu \mathrm{g} / \mathrm{ml})$ and kanamycin $(50 \mu \mathrm{g} / \mathrm{ml})$.
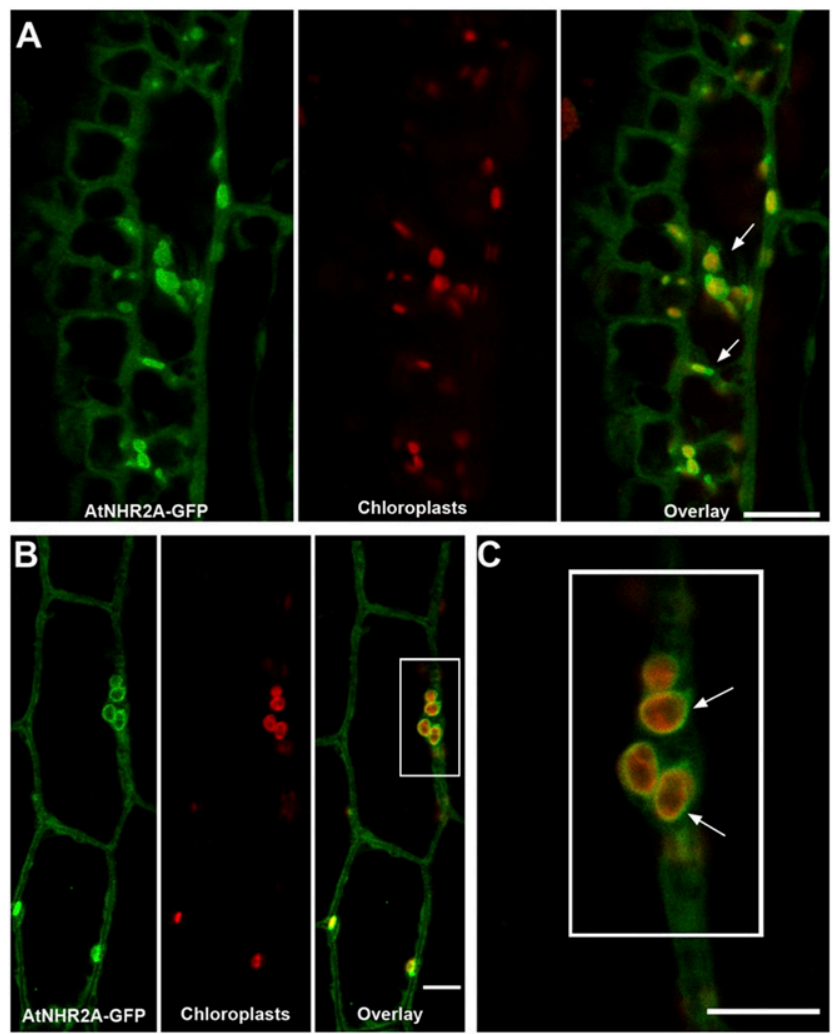

Fig. 9. AtNHR2A-GFP localizes to chloroplasts in Arabidopsis stable transgenic lines. Twelve-day-old seedlings expressing AtNHR2A-GFP in the Atnhr2a mutant background (AtNHR2A-GFP/Atnhr2a-4) were imaged by laser scanning confocal microscopy. White arrows show that the green fluorescent protein (GFP) emission from AtNHR2A-GFP overlaps with chloroplast autofluorescence in $\mathbf{A}$, epidermal cells of cotyledons and $\mathbf{B}$, hypocotyls. C, High-magnification image of the region marked by the white rectangle shows that AtNHR2A-GFP surrounds the periphery of chloroplasts (arrows). Scale bars $=10 \mu \mathrm{m}$.

\section{Plant materials and growth conditions.}

Arabidopsis thaliana T-DNA insertion mutants were obtained via the Arabidopsis Biological Resource Center at The Ohio State University. Lines were genotyped by PCR reactions to confirm homozygosity (O'Malley et al. 2015), using primers listed in Supplementary Table S1. Line SALK_114910 was genotyped using gene-specific primers SALK-114910 RP and SALK114910 LP and T-DNA primer LBb1.3. Line SALK_043140 was genotyped with gene-specific primers SALK043140RP and SALK043140LP and LB1.3. Arabidopsis thaliana seeds were grown in soil in MS (Murashige and Skoog) media, depending on the assay, and were grown for 4 weeks at $21^{\circ} \mathrm{C}$ in a growth chamber, with a 10 -h light and 14-h dark cycle. $N$. benthamiana plants were grown in a growth chamber, under a 10 -h light and $14-\mathrm{h}$ dark cycle, at $25^{\circ} \mathrm{C}$.

\section{Plasmid constructs.}

To generate RFP and GFP fusions, full-length AtNHR2A and $A t N H R 2 B$ were amplified, using cDNA as template, from wildtype Col-0 plants that had been induced with $P$. syringae pv. tabaci for $6 \mathrm{~h}$. Amplified PCR products were cloned by Gateway Technology (Thermo Fisher Scientific Inc, Carlsbad, CA, U.S.A.) into vector pDONR201 to generate entry clones. Entry vectors possessing the AtNHR2A and AtNHR $2 B$ full-length sequence were verified by sequencing and were subsequently transferred to vectors pK7WGR2 (Karimi et al. 2007), pMDC107 and pMDC83 (Curtis and Grossniklaus 2003) to generate 35S::RFP-AtNHR2A, AtNHR2Apro::AtNHR2A-GFP, and $35 S: \because A t N H R 2 B-G F P$, respectively. To generate HAAtNHR2A, AtNHR2A in pDONR201 was transferred to pEarleyGate201 (Earley et al. 2006).

\section{VIGS.}

Agrobacterium tumefaciens GV2260 carrying Tobacco rattle virus (TRV), TRV::NbNHR2 and TRV::00 were grown overnight, on Luria Bertani media supplemented with rifampicin $(25 \mu \mathrm{g} / \mathrm{ml})$ and kanamycin $(50 \mu \mathrm{g} / \mathrm{ml})$, at $28^{\circ} \mathrm{C}$. Overnight cultures were collected by centrifugation and were resuspended in induction medium (10 mM MES, $\mathrm{pH} 5.5,3 \%$ sucrose, $200 \mu \mathrm{M}$ acetosyringone) and were incubated at room temperature with constant shaking for $4 \mathrm{~h}$. Induced cultures containing $T R V$ were mixed with either TRV:00 or TRV ::NHR2 in equal ratios to a final optical density at $600 \mathrm{~nm}\left(\mathrm{OD}_{600}\right)=1$, and these mixtures were infiltrated, using a needleless syringe, into twoweek-old $N$. benthamiana plants. Three weeks after infiltration, NbNHR2-silenced plants and nonsilenced controls were vacuum infiltrated with bacterial pathogens to examine disease symptoms, monitor bacterial multiplication in planta, or to quantify bacterial growth at 0 and 3 dpi.

\section{Pathogen infection assays in A. thaliana.}

Four-week-old plants from genotypes wild-type Col-0, Atnhr2a, Atnhr $2 b$, and Atnhr2bAtnhr $2 a$ plants grown on MS plates were flood-inoculated with the nonadapted pathogen $P$. syringae pv. tabaci or the adapted pathogen $P$. syringae pv. tomato DC3000 at $1 \times 10^{5} \mathrm{CFU} / \mathrm{ml}$ (Ishiga et al. 2011). Inoculated leaves were sampled at 0 and 3 dpi to assess bacterial growth. Disease phenotype was evaluated between 5 and $7 \mathrm{dpi}$.

For syringe-inoculation, 4-to 6-week-old plants were infiltrated with a needleless syringe with $P$. syringae pv. tabaci at $1 \times 10^{5} \mathrm{CFU} / \mathrm{ml}$. Infiltrated leaves were collected at 0 and $3 \mathrm{dpi}$ and leaves were ground in water, were serially diluted, and were plated to enumerate bacterial populations.

\section{Gene expression analysis.}

Downregulation of $N b N H R 2$ was measured by qRT-PCR. RNA was extracted from three independent $N b N H R 2$-silenced 
lines (TRV::NbNHR2) and three independent controls (TRV:: 00). Total RNA was extracted, using Trizol (Thermo Fisher Scientific Inc.), and $2 \mu \mathrm{g}$ of total RNA was treated with DNAse (TurboTM DNase; Thermo Fisher Scientific, Inc.). DNAsetreated RNA was used for cDNA synthesis, using random primers and MultiScribe RT (Thermo Fisher Scientific Inc.). qRT-PCR reaction was performed by mixing $1 \mu \mathrm{l}$ of cDNA with primers with the GoTaq SYBR premix (Promega, Madison, WI, U.S.A.) in a 20- $\mu 1$ final reaction volume. Expression of $N b N H R 2$ in $N b N H R 2$-silenced lines was compared normalized against the expression of actin, used as housekeeping gene, and was compared with the expression of NHR2 in nonsilenced control lines.

To measure expression of AtNHR2A and $A t N H R 2 B$ in Arabidopsis, wild-type Col-0 and the Atnhr2a and Atnhr $2 b$ mutants were grown on MS plates and flood-inoculated with $P$. syringae pv. tabaci at $1 \times 10^{5} \mathrm{CFU} / \mathrm{ml}$ or mock-treated. Above-ground tissues from wild-type Col-0 were harvested at $0,1,6$, and 12 hpi to identify the patterns of AtNHR2A and AtNHR $2 B$ gene expression. Above-ground tissues from wildtype Col-0 and the mutants Atnhr2a and Atnhr2b were harvested at 12 hpi to compare the levels of expression of AtNHR $2 A$ and AtNHR $2 B$ between wild-type Col-O and the mutants. Tissues were collected in liquid nitrogen for RNA extraction followed by cDNA synthesis, as described above. Expression of individual genes was normalized against AtGAPDH (glyceraldehyde 3-phosphate dehydrogenase) (At1g13440) used as housekeeping gene.

\section{Callose deposition.}

Three- to five-week-old wild-type Col-0, Atnhr2a, Atnhr2b, and Atnhr $2 b \quad X$ Atnhr $2 a$ double mutants were syringeinoculated with $P$. syringae pv. tabaci at $1 \times 10^{6} \mathrm{CFU} / \mathrm{ml}$. Control plants were inoculated with water only. Six to nine inoculated leaves were detached from six independent plants for each genotype, after $24 \mathrm{hpi}$, and were stained with $5 \%$ aniline blue to visualize callose deposits (Kvitko et al. 2009). Images were taken by Nikon 90i upright scanning laser confocal microscope (Nikon, Tokyo) using a DAPI (4',6diamidino-2-phenylindole) filter with excitation wavelength of $405 \mathrm{~nm}$ and an emission wavelength of 450 to $510 \mathrm{~nm}$. Callose deposits were quantified by counting deposits in six images per sample taken from random microscopic fields by Image J software.

\section{Confocal microscopy studies to investigate subcellular localization of AtNHR2A and AtNHR2B.}

Plasmid constructs containing AtNHR2Apro::AtNHR2AGFP, 35S::AtNHR2B-GFP, and RFP-AtNHR2A were transformed into Agrobacterium tumefaciens GV2260. To examine the subcellular localization of AtNHR2A and AtNHR2B, overnight cultures of Agrobacterium tumefaciens harboring AtNHR2Apro::AtNHR2A-GFP and 35S::AtNHR2B-GFP were pelleted, were resuspended in induction buffer $(20 \mathrm{mM}$ MES, $\mathrm{pH} 5.5,3 \%$ sucrose, $200 \mu \mathrm{M}$ acetosyringone), and were incubated at room temperature, with constant shaking, for $4 \mathrm{~h}$. Induced cultures were adjusted to an $\mathrm{OD}_{600}=0.3$. Agrobacterium tumefaciens strains harboring AtNHR2Apro:: AtNHR2A-GFP and 35S::AtNHR2B-GFP were infiltrated into fully expanded leaves of 3-week-old $N$. benthamiana plants using a needless syringe. After 2 to 3 days, samples were taken from the infiltrated leaf area and were imaged with a Leica TCS SP8 confocal microscope (Leica Microsystems, Wetzlar, Germany) with sequential scanning mode. Samples were excited using the 488-nm argon laser lines with an emission band of $510 \mathrm{~nm}$ for GFP detection and $685 \mathrm{~nm}$ for chlorophyll autoflourescence detection.
To examine the colocalization of AtNHR2A and AtNHR2B, Agrobacterium tumefaciens GV2260 harboring 35S::RFPAtNHR2A and 35S::AtNHR2B-GFP were coinfiltrated into $N$. benthamiana as described above. Imaging for colocalization studies using 35S::AtNHR2B-GFP and 35S::RFP-AtNHR2A constructs were performed in sequential mode, using the same settings as above for GFP detection and 532-nm argon laser line for RFP excitation.

To examine the localization of AtNHR2A-GFP in stable Arabidopsis transgenic lines, we used the complementing line AtNHR2A-GFP/Atnhr $2 a-4$ for imaging. Seedlings were grown on MS media for 12 days. Seedlings were mounted on glass slides and were imaged with a confocal microscope.

\section{Coimmunoprecipitation assay.}

Agrobacterium tumefaciens containing plasmids AtNHR2BGFP and HA-AtNHR2A were coinfiltrated into $N$. benthamiana for transient coexpression as described above. Free 35S-GFP and HA-AtNHR2A were also coinfiltrated, to be used as negative control. Leaf samples were collected at 3 days after infiltration for protein extraction. Total protein extracts $(1 \mathrm{mg})$ were mixed with $20 \mu \mathrm{l}$ of Pierce HA epitope tag antibody conjugated to agarose beads (Thermo Fisher Scientific) and were incubated overnight at $4^{\circ} \mathrm{C}$ with end-to-end rocking. After incubation, protein complexes bound to beads were washed three times with $1 \times$ Tris-buffered saline $(50 \mathrm{mM}$ Tris- $\mathrm{HCl}, 150 \mathrm{mM} \mathrm{NaCl}, \mathrm{pH}$ 7.5), followed by two more washes with a higher stringency buffer ( $50 \mathrm{mM}$ Tris- $\mathrm{HCl}, 500 \mathrm{mM} \mathrm{NaCl}, \mathrm{pH}$ 7.5). Protein complexes bound to the beads were eluted in $2 \times$ sodium dodecyl sulfate (SDS) protein loading buffer, were loaded and run into a SDS-polyacrylamide electrophoresis gel, and were transferred to nitrocellulose membranes. Proteins were detected by Western blot, by incubating the nitrocellulose membrane with anti-GFPhorseradish peroxidase (HRP) (1:1,000 dilution; Miltenyi Biotec, Cologne, Germany) or anti-HA-HRP antibodies (1:1,000 dilution; Thermo Fisher Scientific Inc.) and detecting by chemiluminescence (Santa Cruz Biotechnology, Santa Cruz, CA, U.S.A.).

\section{ACKNOWLEDGMENTS}

We thank S. Winter and M. A. Parra-Galindo for assistance with statistical analysis and J. Gallaway for plant care. We also thank I. Tzanetakis for helpful advice regarding the phylogenetic analysis, R. Venkategowda for critical reading of the manuscript and all members of the Rojas lab for productive discussions.

\section{LITERATURE CITED}

Alonso, J. M., and Stepanova, A. N. 2003. T-DNA mutagenesis in Arabidopsis. Methods Mol. Biol. 236:177-188.

Boller, T., and Felix, G. 2009. A renaissance of elicitors: Perception of microbe-associated molecular patterns and danger signals by patternrecognition receptors. Annu. Rev. Plant Biol. 60:379-406.

Caplan, J. L., Kumar, A. S., Park, E., Padmanabhan, M. S., Hoban, K. Modla, S., Czymmek, K., and Dinesh-Kumar, S. P. 2015. Chloroplast stromules function during innate immunity. Dev. Cell 34:45-57.

Caplan, J. L., Mamillapalli, P., Burch-Smith, T. M., Czymmek, K., and Dinesh-Kumar, S. P. 2008. Chloroplastic protein NRIP1 mediates innate immune receptor recognition of a viral effector. Cell 132:449-462.

Clay, N. K., Adio, A. M., Denoux, C., Jander, G., and Ausubel, F. M. 2009. Glucosinolate metabolites required for an Arabidopsis innate immune response. Science 323:95-101.

Curtis, M. D., and Grossniklaus, U. 2003. A gateway cloning vector set for high-throughput functional analysis of genes in planta. Plant Physiol 133:462-469.

De Benedictis, M., Brunetti, C., Brauer, E. K., Andreucci, A., Popescu, S. C., Commisso, M., Guzzo, F., Sofo, A., Ruffini Castiglione, M., Vatamaniuk, O. K., and Sanità di Toppi, L. 2018. The Arabidopsis thaliana knockout mutant for phytochelatin synthase 1 (cadl-3) is defective in callose deposition, bacterial pathogen defense and auxin content, but shows an increased stem lignification. Front. Plant Sci. 9:19. 
de Souza, A., Wang, J. Z., and Dehesh, K. 2017. Retrograde signals: Integrators of interorganellar communication and orchestrators of plant development. Annu. Rev. Plant Biol. 68:85-108.

Earley, K. W., Haag, J. R., Pontes, O., Opper, K., Juehne, T., Song, K., and Pikaard, C. S. 2006. Gateway-compatible vectors for plant functional genomics and proteomics. Plant J. 45:616-629.

Emanuelsson, O., Nielsen, H., Brunak, S., and von Heijne, G. 2000. Predicting subcellular localization of proteins based on their N-terminal amino acid sequence. J. Mol. Biol. 300:1005-1016.

Emanuelsson, O., Nielsen, H., and von Heijne, G. 1999. ChloroP, a neural network-based method for predicting chloroplast transit peptides and their cleavage sites. Protein Sci. 8:978-984.

Enrique, R., Siciliano, F., Favaro, M. A., Gerhardt, N., Roeschlin, R., Rigano, L., Sendin, L., Castagnaro, A., Vojnov, A., and Marano, M. R. 2011. Novel demonstration of RNAi in citrus reveals importance of citrus callose synthase in defence against Xanthomonas citri subsp. citri. Plant Biotechnol. J. 9:394-407.

Fujimoto, M., and Ueda, T. 2012. Conserved and plant-unique mechanisms regulating plant post-Golgi traffic. Front. Plant Sci. 3:197.

Galletti, R., Denoux, C., Gambetta, S., Dewdney, J., Ausubel, F. M., De Lorenzo, G., and Ferrari, S. 2008. The AtrbohD-mediated oxidative burst elicited by oligogalacturonides in Arabidopsis is dispensable for the activation of defense responses effective against Botrytis cinerea. Plant Physiol. 148:1695-1706.

Geldner, N., Dénervaud-Tendon, V., Hyman, D. L., Mayer, U., Stierhof, Y. D., and Chory, J. 2009. Rapid, combinatorial analysis of membrane compartments in intact plants with a multicolor marker set. Plant J. 59:169-178.

Heath, M. C. 2000. Nonhost resistance and nonspecific plant defenses. Curr. Opin. Plant Biol. 3:315-319.

Ishiga, Y., Ishiga, T., Uppalapati, S. R., and Mysore, K. S. 2011. Arabidopsis seedling flood-inoculation technique: A rapid and reliable assay for studying plant-bacterial interactions. Plant Methods 7:32.

Ishiga, Y., Watanabe, M., Ishiga, T., Tohge, T., Matsuura, T., Ikeda, Y., Hoefgen, R., Fernie, A. R., and Mysore, K. S. 2017. The SAL-PAP chloroplast retrograde pathway contributes to plant immunity by regulating glucosinolate pathway and phytohormone signaling. Mol. Plant-Microbe Interact 30:829-841.

Jacobs, A. K., Lipka, V., Burton, R. A., Panstruga, R., Strizhov, N., SchulzeLefert, P., and Fincher, G. B. 2003. An Arabidopsis callose synthase, GSL5, is required for wound and papillary callose formation. Plant Cell 15:2503-2513.

Jones, J. D., and Dangl, J. L. 2006. The plant immune system. Nature 444: 323-329.

Karimi, M., Depicker, A., and Hilson, P. 2007. Recombinational cloning with plant gateway vectors. Plant Physiol. 145:1144-1154.

Khan, M., Subramaniam, R., and Desveaux, D. 2016. Of guards, decoys, baits and traps: Pathogen perception in plants by type III effector sensors. Curr. Opin. Microbiol. 29:49-55.

Kvitko, B. H., Park, D. H., Velásquez, A. C., Wei, C. F., Russell, A. B., Martin, G. B., Schneider, D. J., and Collmer, A. 2009. Deletions in the repertoire of Pseudomonas syringae pv. tomato DC3000 type III secretion effector genes reveal functional overlap among effectors. PLoS Pathog. 5:e1000388.

Lee, H. A., Lee, H. Y., Seo, E., Lee, J., Kim, S. B., Oh, S., Choi, E., Choi, E., Lee, S. E., and Choi, D. 2017. Current understandings of plant nonhost resistance. Mol. Plant-Microbe Interact 30:5-15.

Lee, S., Whitaker, V. M., and Hutton, S. F. 2016. Mini review: Potential applications of non-host resistance for crop improvement. Front. Plant Sci. 7:997.

Leister, D. 2012. Retrograde signaling in plants: From simple to complex scenarios. Front. Plant Sci. 3:135.

Luna, E., Pastor, V., Robert, J., Flors, V., Mauch-Mani, B., and Ton, J. 2011. Callose deposition: A multifaceted plant defense response. Mol. PlantMicrobe Interact 24:183-193.

Moore, I., and Murphy, A. 2009. Validating the location of fluorescent protein fusions in the endomembrane system. Plant Cell 21:1632-1636.

Nelson, B. K., Cai, X., and Nebenführ, A. 2007. A multicolored set of in vivo organelle markers for co-localization studies in Arabidopsis and other plants. Plant J. 51:1126-1136.
Nishimura, M. T., Stein, M., Hou, B. H., Vogel, J. P., Edwards, H., and Somerville, S. C. 2003. Loss of a callose synthase results in salicylic acid-dependent disease resistance. Science 301:969-972.

Nomura, H., Komori, T., Uemura, S., Kanda, Y., Shimotani, K., Nakai, K., Furuichi, T., Takebayashi, K., Sugimoto, T., Sano, S., Suwastika, I. N., Fukusaki, E., Yoshioka, H., Nakahira, Y., and Shiina, T. 2012 Chloroplast-mediated activation of plant immune signalling in Arabidopsis. Nat. Commun. 3:926.

O’Malley, R. C., Barragan, C. C., and Ecker, J. R. 2015. A user's guide to the Arabidopsis T-DNA insertion mutant collections. Methods Mol. Biol. 1284:323-342.

Petsalaki, E. I., Bagos, P. G., Litou, Z. I., and Hamodrakas, S. J. 2006. PredSL: A tool for the N-terminal sequence-based prediction of protein subcellular localization. Genomics Proteomics Bioinformatics 4:48-55.

Rojas, C. M., Senthil-Kumar, M., Wang, K., Ryu, C. M., Kaundal, A., and Mysore, K. S. 2012. Glycolate oxidase modulates reactive oxygen species-mediated signal transduction during nonhost resistance in Nicotiana benthamiana and Arabidopsis. Plant Cell 24:336-352.

Saitou, N., and Nei, M. 1987. The neighbor-joining method: A new method for reconstructing phylogenetic trees. Mol. Biol. Evol. 4:406-425.

Schwacke, R., Fischer, K., Ketelsen, B., Krupinska, K., and Krause, K. 2007. Comparative survey of plastid and mitochondrial targeting properties of transcription factors in Arabidopsis and rice. Mol. Genet. Genomics 277:631-646.

Schwacke, R., Schneider, A., van der Graaff, E., Fischer, K., Catoni, E., Desimone, M., Frommer, W. B., Flügge, U. I., and Kunze, R. 2003 ARAMEMNON, a novel database for Arabidopsis integral membrane proteins. Plant Physiol. 131:16-26.

Senthil-Kumar, M., and Mysore, K. S. 2013. Nonhost resistance against bacterial pathogens: Retrospectives and prospects. Annu. Rev. Phytopathol. 51:407-427.

Serrano, I., Audran, C., and Rivas, S. 2016. Chloroplasts at work during plant innate immunity. J. Exp. Bot. 67:3845-3854.

Sun, X., Feng, P., Xu, X., Guo, H., Ma, J., Chi, W., Lin, R., Lu, C., and Zhang, L. 2011. A chloroplast envelope-bound PHD transcription factor mediates chloroplast signals to the nucleus. Nat. Commun. 2:477.

Ton, J., and Mauch-Mani, B. 2004. $\beta$-amino-butyric acid-induced resistance against necrotrophic pathogens is based on ABA-dependent priming for callose. Plant J. 38:119-130.

Toruño, T. Y., Stergiopoulos, I., and Coaker, G. 2016. Plant-pathogen effectors: Cellular probes interfering with plant defenses in spatial and temporal manners. Annu. Rev. Phytopathol. 54:419-441.

Tsuda, K., and Katagiri, F. 2010. Comparing signaling mechanisms engaged in pattern-triggered and effector-triggered immunity. Curr. Opin. Plant Biol. 13:459-465.

Vogel, J., and Somerville, S. 2000. Isolation and characterization of powdery mildew-resistant Arabidopsis mutants. Proc. Natl. Acad. Sci. U.S.A. 97:1897-1902.

Wang, K., Kang, L., Anand, A., Lazarovits, G., and Mysore, K. S. 2007. Monitoring in planta bacterial infection at both cellular and whole-plant levels using the green fluorescent protein variant GFPuv. New Phytol. $174: 212-223$

Wang, K., Senthil-Kumar, M., Ryu, C. M., Kang, L., and Mysore, K. S. 2012. Phytosterols play a key role in plant innate immunity against bacterial pathogens by regulating nutrient efflux into the apoplast. Plant Physiol. 158:1789-1802.

Winter, D., Vinegar, B., Nahal, H., Ammar, R., Wilson, G. V., and Provart, N. J. 2007. An "electronic fluorescent pictograph" browser for exploring and analyzing large-scale biological data sets. PLoS One 2: e718.

Yi, S. Y., Shirasu, K., Moon, J. S., Lee, S. G., and Kwon, S. Y. 2014. The activated SA and JA signaling pathways have an influence on flg22triggered oxidative burst and callose deposition. PLoS One 9:e88951.

\section{AUTHOR-RECOMMENDED INTERNET RESOURCE}

Image J software: http://rsb.info.nih.gov/ij 